\title{
Cubefree words with many squares
}

\author{
James Currie $\|^{\mid t}$ and Narad Rampersad" \\ Department of Mathematics and Statistics, University of Winnipeg \\ 515 Portage Avenue, Winnipeg, Manitoba R3B 2E9 (Canada) \\ $\{j$.currie, n.rampersad\}@uwinnipeg.ca
}

received November 26, 2008, revised April 25, 2010, accepted April 28, 2010.

We construct infinite cubefree binary words containing exponentially many distinct squares of length $n$. We also show that for every positive integer $n$, there is a cubefree binary square of length $2 n$.

Keywords: cubefree word, square

\section{Introduction}

A square is a non-empty word of the form $x x$, and a cube is a non-empty word of the form $x x x$. An overlap is a word of the form axaxa, where $a$ is a letter and $x$ is a word (possibly empty). A word is squarefree (resp. cubefree, overlap-free) if none of its factors are squares (resp. cubes, overlaps). For further background material concerning combinatorics on words we refer the reader to [2].

It is well-known that there exist infinite squarefree words over a ternary alphabet and infinite overlapfree words over a binary alphabet. Clearly, any overlap-free word is also cubefree. Any infinite cubefree binary word must contain squares; however, Dekking [9] proved that there exists an infinite cubefree binary word containing no squares $x x$ where the length of $x$ is greater than 3 (see also [14, 15]). In this paper we consider instead the existence of infinite cubefree binary words with many distinct squares.

Most known constructions of infinite cubefree words involve the iteration of a morphism. In the early 80's, Berstel [3] revitalized the study of the construction of words avoiding repetitions by the iteration of morphisms. Words constructed in this manner are often refered to as infinite DOL words. Ehrenfeucht and Rozenberg [10, 11, 12] proved several results concerning the factor complexity of infinite D0L words. They showed that any squarefree or cubefree D0L word has $O(n \log n)$ factors of length $n$. Thus, an infinite cubefree D0L word cannot have many distinct square factors. By constrast, we show here how to construct infinite cubefree binary words containing exponentially many distinct squares of length $n$.

Other work related to the problems considered here include [1, 7, 8].

Let $\mu$ denote the Thue-Morse morphism: i.e., the morphism that maps $0 \rightarrow 01$ and $1 \rightarrow 10$. The Thue-Morse word is the infinite word

$$
\mathbf{t}=011010011001011010010110 \cdots
$$

\footnotetext{
${ }^{\dagger}$ The first author is supported by an NSERC Discovery Grant.

¥The second author is supported by an NSERC Postdoctoral Fellowship. 
obtained by iteratively applying $\mu$ to the word 0 . The Thue-Morse word is well-known to be overlap-free, and hence, a fortiori, cubefree [17]. The squares occurring in the Thue-Morse word were characterized by Pansiot [13] and Brlek [5] as follows. Define sets $A=\{00,11,010010,101101\}$ and

$$
\mathcal{A}=\bigcup_{k \geq 0} \mu^{k}(A)
$$

The set $\mathcal{A}$ is the set of squares appearing in the Thue-Morse word.

Shelton and Soni [16] characterized the overlap-free squares (the result is also attributed to Thue by Berstel [4]), as being the conjugates of the words in $\mathcal{A}$. (A conjugate of $x$ is a word $y$ such that $x=u v$ and $y=v u$ for some $u, v$.) Currie and Rampersad [7] showed that the conjugates of the words in $\mathcal{A}$ are also precisely the $7 / 3$-power-free squares. Thus, there are only $7 / 3$-power-free squares of length $2 n$ when $n$ is a power of 2 , or 3 times a power of 2 . By contrast, we show that there are cubefree binary squares of length $2 n$ for every positive integer $n$. We use this result to construct infinite cubefree binary words containing exponentially many distinct squares.

\section{Main results}

The main results of this paper are the following two theorems.

Theorem 1 Let $n$ be a positive integer. There exists a cubefree binary square of length $2 n$.

Theorem 2 There exists an infinite cubefree binary word containing exponentially many distinct squares of length $n$.

We first establish some preliminary results.

Lemma 3 The Thue-Morse word contains a factor of the form $x=1001 x^{\prime \prime}=x^{\prime} 1001$ of every positive even length $n \neq 2,6$.

Proof: Aberkane and Currie [1, Lemma 4] proved that for every integer $m \geq 6$, the Thue-Morse word contains a factor of length $m$ of the form $10 y 10$. Then the Thue-Morse word also contains the factor $\mu(10 y 10)=1001 \mu(y) 1001$, which has length $2 m$. Finally, we observe that 10011001 and 1001101001 are factors of the Thue-Morse word of lengths 8 and 10 respectively.

Lemma 4 If $y$ is overlap-free and ayb is a cube of period $p$, then $p \leq|a b|$.

Proof: Otherwise deleting $a$ and $b$ removes less than a full period from $a y b$, leaving an overlap.

Lemma 5 If $z$ is a factor of yyy where $|y|=p$ and $|z| \leq p+1$, then there are two occurrences of $z$ in yyy.

Proof: Certainly if $z$ is a factor of $y y$ it occurs twice in yyy. If $\mathrm{z}$ is a factor of yyy but not of $y y$, then $z$ must span the central $y$ of $y y y$ and a bit more on both ends, giving $z$ a length of $p+2$ or more.

Theorem 6 Let $x$ be a factor of the Thue-Morse word of the form $x=1001 x^{\prime \prime}=x^{\prime} 1001$. Then the word $x 0 x 0$ is cubefree. 
Remark 1 Word 01010 occurs exactly once in $x 0 x 0$. (Note that this word is an overlap, and hence not a factor of the Thue-Morse word.)

Proof of Theorem 6: Suppose yyy is a cube in $x 0 x 0$ with $|y|=p>0$.

Case 1: Period $p \geq 4$.

By Lemma 5 and Remark 1, word 01010 is not a factor of yyy. We have two possibilities:

(a) Cube yyy is a factor of $x^{\prime} 100101$. This is impossible by Lemma 4, since $x^{\prime} 1001$ is overlapfree, $|01|=2$, and $p \geq 4>2$.

(b) Cube yyy is a factor of $101001 x^{\prime \prime} 0$. This is again impossible by Lemma 4 since $1001 x^{\prime \prime}$ is overlap-free.

Case 2: Period $p \leq 3$.

If 01010 is a factor of $y y y$, then one of 001010 and 010100 is a factor. However, neither of these has period 1, 2 or 3; this is impossible. We conclude that 01010 is not a factor of yyy. This gives a similar case breakdown as in Case 1.

(a) Cube yyy is a factor of $x^{\prime} 100101$.

(i) Cube yyy is a suffix of $x^{\prime} 100101$. In this case, $p \leq 2$ by Lemma 4, since $x^{\prime} 1001$ is overlap-free. However, the longest suffix of $x^{\prime} 100101$ of period 1 or 2 is 0101 , which is cubefree.

(ii) Cube yyy is a suffix of $x^{\prime} 10010$. This forces $p=1$, which is impossible.

(b) Cube yyy is a factor of $101001 x^{\prime \prime} 0$.

(i) Cube yyy is a prefix of $101001 x^{\prime \prime} 0$ or of $01001 x^{\prime \prime} 0$. If $x^{\prime \prime}$ is the empty word, then $x 0 x 0=1001010010$ is clearly cubefree, so let us assume that $\left|x^{\prime \prime}\right| \geq 4$. Since $|y y y|=$ $3 p \leq 9 \leq\left|01001 x^{\prime \prime}\right|$, yyy is a factor of $101001 x^{\prime \prime}$. This is symmetrical to Case $2 \mathrm{a}$.

(ii) Cube yyy is a factor of $1001 x^{\prime \prime} 0=x 0$. This is impossible by Case $2 \mathrm{a}$.

Theorem 7 Let $x$ be a factor of the Thue-Morse word of the form $x=1001 x^{\prime \prime}=x^{\prime} 1001$. Then the word $x 101100 x 101100$ is cubefree.

Remark 2 Word 00100 occurs exactly once in $x 101100 x 101100$. Word 11011 occurs exactly twice.

Proof of Theorem 7; Suppose $y y y$ is a cube in $x 101100 x 101100$ with $|y|=p>0$.

Case 1: Period $p \geq 4$.

By Lemma 5 and Remark 2, word 00100 is not a factor of yyy. We have two possibilities:

(a) Cube yyy is a factor of $x 10110010$.

Word $x 10110010$ contains 11011 as a factor exactly once. By Lemma 5] and Remark 2, there are two possibilities:

(i) Cube yyy is contained in $x 101$.

In this case, $p \leq 3$ by Lemma 4 , since $x$ is overlap-free. This is a contradiction. 
(ii) Cube yyy is contained in 10110010.

This is clearly impossible.

(b) Cube yyy is a factor of $0 x 101100$.

Again, word $0 x 101100$ contains 11011 as a factor exactly once. Therefore, either yyy is contained in 101100 or in $0 x 101$. The first alternative evidently is impossible, while the second is ruled out by Lemma 4 .

Case 2: Period $p \leq 3$.

If 00100 is a factor of yyy, then we must have $p=3$, since 00100 does not have period 1 or 2. However, in $x 101100 x 101100$, the maximal factor of period 3 containing 00100 is 1001001 , which is not a cube. We conclude that 00100 is not a factor of yyy. This gives a similar case breakdown to Case 1

(a) Cube yyy is a factor of $x 10110010$.

By Lemma 4 the word $x 10$ must be cubefree. Therefore, yyy must be a suffix of one of these words:

$$
\begin{aligned}
& w_{8}=x^{\prime} 100110110010 \\
& w_{7}=x^{\prime} 10011011001 \\
& w_{6}=x^{\prime} 1001101100 \\
& w_{5}=x^{\prime} 100110110 \\
& w_{4}=x^{\prime} 10011011 \\
& w_{3}=x^{\prime} 1001101
\end{aligned}
$$

None of the $w_{n}$ ends in a cube of period 1, 2 or 3 . (In the case of words $w_{4}, w_{3}$, the longest suffixes of period 3 have lengths 6 and 5 respectively.) It follows that yyy is not a suffix of any of the $w_{n}$, and this case does not occur.

(b) Cube yyy is a factor of $0 x 101100$.

Since $|y y y|=3 p \leq 9 \leq|0 x|$, yyy is a factor of $0 x$ or of $x 101100$. The first possibility was ruled out in the proof of Theorem 6 , and the second in Case $2 \mathrm{a}$

Theorems 6 and 7 together establish Theorem 1 Next we show that the number of cubefree binary squares of length $n$ grows exponentially.

Proposition 8 There exist exponentially many cubefree binary squares of length $n$.

Proof: Let $m$ be a positive integer and let $x x$ be a cubefree binary square of length $2 m$ over $\{0,1\}$. Suppose that 0 occurs at least as often as 1 in $x$. Construct a new cubefree square $y y$ over $\{0,1,2\}$, where $y$ is obtained from $x$ by arbitrarily replacing some of the 0 's in $x$ by 2 's. There are at least $2^{m / 2}$ such squares $y y$ of length $2 m$.

Let $h$ be the morphism

$$
\begin{aligned}
& 0 \rightarrow 001011 \\
& 1 \rightarrow 001101 \\
& 2 \rightarrow 011001
\end{aligned}
$$


Brandenburg [6, Theorem 6] showed that $h$ maps cubefree words to cubefree words. Moreover, since $h$ is uniform and injective, the set of words $h(y y)$ consists of at least $2^{m / 2}$ cubefree squares of length $12 m$. Asymptotically, we thus have exponentially many cubefree binary squares of length $n$, as required.

We now prove Theorem2

Proof of Theorem 2: In the proof of Proposition 8 we showed that there are at least $2^{m / 2}$ cubefree binary squares of length $12 m$ for every positive integer $m$. Let $S$ therefore be any set of cubefree squares over $\{0,1\}$ where $S$ contains at least $2^{m / 2}$ words of length $12 m$ for every positive integer $m$. Let $\mathbf{x}=x_{1} x_{2} \ldots$ be any infinite cubefree binary word over $\{2,3\}$. Construct a word

$$
\mathbf{w}=x_{1} S_{1} x_{2} S_{2} \cdots,
$$

where the set of $S_{i}$ 's is equal to the set $S$, so that w is cubefree and contains exponentially many distinct squares of length $n$. Let $g$ be the morphism

$$
\begin{aligned}
& 0 \rightarrow 001001101 \\
& 1 \rightarrow 001010011 \\
& 2 \rightarrow 001101011 \\
& 3 \rightarrow 011001011 .
\end{aligned}
$$

Brandenburg [6, Theorem 6] showed that $g$ maps cubefree words to cubefree words. Thus, $g(\mathbf{w})$ is cubefree and, by the uniformity and injectivity of $g$, contains exponentially many distinct squares of length $n$.

Note that Theorem 2 implies that existence of an infinite cubefree binary word with exponential factor complexity-i.e., with exponentially many factors of length $n$. Similarly, one can easily construct an infinite squarefree word over $\{0,1,2\}$ with exponential factor complexity.

Proposition 9 There exists an infinite squarefree word over $\{0,1,2\}$ with exponential factor complexity.

Proof: Let $\mathbf{w}$ be any infinite squarefree word over $\{0,1,2\}$ and let $\mathbf{x}$ be any infinite word over $\{3,4\}$ with $2^{n}$ factors of length $n$ for every positive $n$. Let $\mathbf{y}$ be the word obtained by forming the perfect shuffle of $\mathbf{w}$ and $\mathbf{x}$ : that is, if $\mathbf{w}=w_{0} w_{1} w_{2} \cdots$ and $\mathbf{x}=x_{0} x_{1} x_{2} \cdots$, then define $\mathbf{y}=w_{0} x_{0} w_{1} x_{1} w_{2} x_{2} \cdots$. Clearly, $\mathbf{y}$ is a squarefree word with exponential factor complexity. Let $f$ be the morphism

$$
\begin{aligned}
& 0 \rightarrow 010201202101210212 \\
& 1 \rightarrow 010201202102010212 \\
& 2 \rightarrow 010201202120121012 \\
& 3 \rightarrow 010201210201021012 \\
& 4 \rightarrow 010201210212021012 .
\end{aligned}
$$

Brandenburg [6, Theorem 4] showed that $f$ maps squarefree words to squarefree words. The uniformity and injectivity of $f$ implies that $f(\mathbf{y})$ is a squarefree word with exponential factor complexity, as required. 


\section{References}

[1] A. Aberkane, J. Currie, "There exist binary circular $5 / 2^{+}$power free words of every length", Electron. J. Combinatorics 11 (2004), \#R10.

[2] J.-P. Allouche, J. Shallit, Automatic Sequences: Theory, Applications, Generalizations, Cambridge, 2003.

[3] J. Berstel, "Mots sans carré et morphismes itérés”, Discrete Math. 29 (1980), 235-244.

[4] J. Berstel, "Axel Thue's work on repetitions in words". In P. Leroux, C. Reutenauer, eds., Séries formelles et combinatoire algébrique, Publications du LaCIM, pp 65-80, UQAM, 1992.

[5] S. Brlek, "Enumeration of factors in the Thue-Morse word", Discrete Appl. Math. 24 (1989), 83-96.

[6] F.-J. Brandenburg, "Uniformly growing $k$ th power-free homomorphisms", Theoret. Comput. Sci. 23 (1983), 69-82.

[7] J. Currie, N. Rampersad, "Infinite words containing squares at every position", Theor. Inform. Appl. 44 (2010), 113-124.

[8] J. Currie, N. Rampersad, J. Shallit, "Binary words containing infinitely many overlaps", Electron. J. Combinatorics 13 (2006), \#R82.

[9] F. M. Dekking, "On repetitions of blocks in binary sequences", J. Combin. Theory. Ser. A 20 (1976), 292-299.

[10] A. Ehrenfeucht, G. Rozenberg, "On the subword complexity of square-free DOL languages", Theoret. Comput. Sci. 16 (1981), 25-32.

[11] A. Ehrenfeucht, G. Rozenberg, "On the subword complexity of $m$-free DOL languages", Inform. Process. Lett. 17 (1983), 121-124.

[12] A. Ehrenfeucht, G. Rozenberg, "On the size of the alphabet and the subword complexity of squarefree DOL languages”, Semigroup Forum 26 (1983), 215-223.

[13] J.-J. Pansiot, “The Morse sequence and iterated morphisms”, Inform. Process. Lett. 12 (1981), 6870.

[14] N. Rampersad, J. Shallit, M.-w. Wang, "Avoiding large squares in infinite binary words", Theoret. Comput. Sci. 339 (2005), 19-34.

[15] J. Shallit, "Simultaneous avoidance of large squares and fractional powers in infinite binary words", Int'l. J. Found. Comput. Sci. 15 (2004), 317-327.

[16] R. Shelton, R. Soni, “Chains and fixing blocks in irreducible sequences”, Discrete Math. 54 (1985), 93-99.

[17] A. Thue, “Über die gegenseitige Lage gleicher Teile gewisser Zeichenreihen”, Kra. Vidensk. Selsk. Skrifter. I. Math. Nat. Kl. 1 (1912), 1-67. 